\title{
High burden of self-reported sexually transmitted infections among key populations in Mozambique: the urgent need for an integrated surveillance system
}

Makini A. S. Boothe ${ }^{1 *}$ (D), Charlotte Comé2 ${ }^{2}$ Cynthia Semá Baltazar ${ }^{1,2}$, Noela Chicuecue $^{3}$, Jessica Seleme 3 , Denise Chitsondzo Langa ${ }^{2}$, Isabel Sathane ${ }^{3}$, Henry F. Raymond ${ }^{4}$, Erika Fazito ${ }^{5}$, Marleen Temmerman ${ }^{1,6}$ and Stanley Luchters ${ }^{1,7,8,9}$

\begin{abstract}
Background: Key populations - men who have sex with men (MSM), female sex workers (FSW) and people who inject drugs (PWID) - are at high risk for sexually transmitted infections (STI) given their sexual risk behaviours along with social, legal and structural barriers to prevention, care and treatment services. The purpose of this secondary analysis is to assess the prevalence of self-reported STIs and to describe associated risk factors among participations of the first Biological Behavioural Surveillance (BBS) in Mozambique.

Methods: Responses from the first BBS surveys conducted in 2011-2014 were aggregated across survey-cities to produce pooled estimates for each population. Aggregate weighted estimates were computed to analyse selfreported STI prevalence. Unweighted pooled estimates were used in multivariable logistic regression to identify risk factors associated with self-reported STI.

Results: The prevalence of self-reported STI was 11.9\% (95\% Cl, 7.8-16.0), 33.6\% (95\% Cl, 29.0-41.3), and 22.0\% (95\% $\mathrm{Cl}, 17.0-27.0)$ among MSM, FSW and PWID, respectively. MSM who were circumcised, had HIV, reported drug use, reported receptive anal sex, and non-condom use with their last male partner had greater odds of STI self-report. STI-self report among FSW was associated with living in Beira, being married, employment aside from sex work, physical violence, sexual violence, drug use, access to comprehensive HIV prevention services, non-condom use with last client, and sexual relationship with a non-client romantic partner. Among PWID, risk factors for selfreported STI included living in Nampula/Nacala, access to HIV prevention services, and sex work.
\end{abstract}

Conclusion: The high-burden of STIs among survey participants requires integrated HIV and STI prevention, treatment, and harm reduction services that address overlapping risk behaviours, especially injection drug use and sex work. A robust public health response requires the creation of a national STI surveillance system for better screening and diagnostic procedures within these vulnerable populations.

\footnotetext{
* Correspondence: makini.boothe@ugent.be; makini.boothe@gmail.com

${ }^{1}$ Faculty of Medicine and Health Sciences, Ghent University, Ghent, Belgium

Full list of author information is available at the end of the article
}

C C The Author(s). 2020 Open Access This article is licensed under a Creative Commons Attribution 4.0 International License, which permits use, sharing, adaptation, distribution and reproduction in any medium or format, as long as you give appropriate credit to the original author(s) and the source, provide a link to the Creative Commons licence, and indicate if changes were made. The images or other third party material in this article are included in the article's Creative Commons licence, unless indicated otherwise in a credit line to the material. If material is not included in the article's Creative Commons licence and your intended use is not permitted by statutory regulation or exceeds the permitted use, you will need to obtain permission directly from the copyright holder. To view a copy of this licence, visit http://creativecommons.org/licenses/by/4.0/ The Creative Commons Public Domain Dedication waiver (http://creativecommons.org/publicdomain/zero/1.0/) applies to the data made available in this article, unless otherwise stated in a credit line to the data. 


\section{Background}

The World Health Organization (WHO) estimates that there are more than one million new cases of curable sexually transmitted infections (STIs) every day globally [1]. These infections - caused by Chlamydia trachomatis, Neisseria gonorrhoeae, Treponema pallidum, and Trichomonas vaginalis - can have a serious impact on health status including cervical cancer, pelvic inflammatory disease, infertility and adverse mental health [2, 3]. STIs can also be transmitted through mother-tochild transmission with adverse health outcomes such as stillbirth, neonatal death, low-birth-weight and prematurity, sepsis, pneumonia, neonatal conjunctivitis, and congenital deformities [2]. STIs can also dramatically increase the risk of acquiring Human immunodeficiency virus (HIV) [1]. In the most recent AIDS Indicator Survey conducted in Mozambique, selfreported STIs among the general adult population, aged 15-49, was estimated at $7 \%$ among women and $5 \%$ on men [4].

Key populations (KP), defined as men who have sex with men (MSM), female sex workers (FSW) and people who inject drugs (PWID), are vulnerable to both HIV and other STIs given their high-risk sexual and drug use behaviours, further heightened by structural barriers such as low access to quality health services, stigma and discrimination [3]. Given, their risk profile, WHO advocates for STI interventions to be targeted to these groups [5]. The WHO focuses on four STIs that are curable: syphilis, gonorrhoea, chlamydia and trichomoniasis [1]. However, in Mozambique, similar to other low- and middle-income countries, diagnostic tests are largely unavailable and STI case management is based on symptomology $[2,6]$.

Mozambique's National HIV Strategic Plans (20102014, 2015-2019) identify STI screening as an important intervention for people living with $\operatorname{HIV}[7,8]$. Given the lack of data about KP, the Strategic Plans also called for special surveys to be conducted among MSM, FSW, and PWID to estimate HIV prevalence, assess risk factors for HIV infection, and estimate population size of these KP. The first round of bio-behavioural surveillance surveys (BBS) were conducted between 2011 and 2014 in three urban areas in Mozambique and the methodology and main results have been previously published [9-14]. Prior to the implementation of the BBS surveys, there was no data about STIs in Mozambique among KP. However, given the associated morbidity and mortality, coupled with the social, legal and structural barriers to uptake of health care services among these population groups, it is imperative that STIs are monitored and treated in order to promote general health and wellbeing within these populations. In addition, efforts to control STIs among KP can also reduce transmission among members of the general population who are sexual partners of KP [2].

In this context, the purpose of this secondary analysis is to assess the prevalence of self-reported STIs among participants of the first BBS surveys in Mozambique and to describe risk factors associated with STIs among these three KP groups. This analysis provides an opportunity to assess access to comprehensive HIV/STI services and to monitor the implementation of efforts aimed toward reducing STIs among KP in Mozambique. Finally, the information will provide a baseline and evidence for improving STI prevention, diagnosis and treatment services in Mozambique.

\section{Methods}

\section{Survey design}

The first round of BBS surveys among KPs in Mozambique were conducted in three urban centres Maputo (MSM, FSW, PWID), Beira (MSM, FSW), Nampula (FSW) and Nampula/Nacala (MSM, PWID) - using respondent-driven sampling (RDS) $[15,16]$. RDS is a probability-based peer-to-peer sampling strategy used among hard-to-reach populations. Based on social network size, weights can be computed to produce adjusted estimates representative of the target population in the geographical location where the survey is conducted. The study design for each of the surveys have been previously published and include a description of efforts to reduce bias during data collection, analysis and interpretation [9-14].

\section{Study population}

Participants in the MSM survey were eligible for the survey if they were biologically male, at least 18 years of age, and had engaged in oral or anal sex with one or more men in the 12 months preceding the survey. Being biologically female, at least 15 years of age, and having received money in exchange for sex from someone other than a steady partner in the six months preceding the survey were required of FSW participants. Finally, eligibility criteria for PWID was not restricted by sex, but required an individual to be at least 18 years of age. All individuals who participated in the PWID survey prior to December 2013 must have injected drugs without a prescription in the 12 months preceding the survey, however, due to slow recruitment patterns, this criterion was later modified (from January 2014 onwards) to include any person who had ever injected drugs without a prescription.

All eligible participants in the three surveys needed to have lived, worked or socialized in one of the recruitment areas in the six months preceding the survey, received a valid referral coupon from a peer, and had not previously participated in the study. Participants 
provided separate written informed consent for both the behavioural questionnaire and biological testing; however, only consent to the behavioural questionnaire was necessary in order to be enrolled in the study. Recruitment lasted from July to November 2011 (MSM), September 2011 to March 2012 (FSW) and October 2013 to March 2014 (PWID).

\section{Study measures}

The questionnaires for the three surveys have been published [12-14]. For the purpose of this analysis, HIV is considered separate from STIs because of the emphasis on treatable infections, consistent with WHO guidelines $[1,5]$. Given the lack of laboratory confirmatory testing, questions about self-reported STI symptoms are considered a proxy for possible STI, in line with guidance for biobehavioural surveys among KP [17, 18]. Self-reported STI was defined as responding "yes" to one or more of the following questions: "During the last six months, have you had an abnormal discharge from your vagina, anus or penis?", "During the last six months, have you had a sore or ulcer near your vagina, anus or penis?," and "In the last six months, did someone inform you that you had or could have a sexually transmitted infection?"

\section{Statistical analysis}

RDS-adjusted self-reported STI prevalence was computed for each population by survey city. Due to low sample size, estimates were then pooled to produce an aggregate estimate of the variable of interest for the KP group using the aggregate estimate function of RDS Analyst software [19]. RDS-weights were calculated using the RDS II estimator, which uses the individual network size to create sampling weights [20]. Unweighted pooled estimates were used to conduct bivariable and multivariable logistic regression to identify the correlates associated with the primary outcome of interest: STI self-report. Correlates included in the regression models were selected based on literature review; variables were also included in the model if $p<0.10$ in the bivariate association, while the final model included variables significant at $p<0.05$. Categories of analysis included demographic characteristics, sexual-risk behaviours, HIV status, circumcision (MSM and male PWID), drug use behaviours (not exclusive to injection for MSM and FSW), access to comprehensive HIV prevention services, stigma/discrimination and past experiences with physical or sexual violence (rape). Descriptive analysis for aggregate estimates was conducted using RDS-Analyst [19] and logistic regression was conducted using SAS version 9.4 (SAS Institute, Cary, NC, USA).

\section{Results}

Table 1 presents the unweighted pooled demographic characteristics of the survey participant. There were 1432 MSM, 1240 FSW and 492 PWID enrolled in the surveys distributed across the three cities as follows: Maputo (MSM: 34.6\%, FSW: 32.2\%, PWID: 71.8\%), Beira (MSM: 40.7\%, FSW: 33.2\%), Nampula/Nacala (MSM: 24.7\%, FSW: 34.6\%, PWID: 28.3\%). Male PWID made up the overwhelming majority of the sample population (94.9\%). The majority of MSM and FSW were less than 24 years of age, at 79.2 and $71.6 \%$, respectively, while only $18.7 \%$ of PWID were young. Most survey participants were single or never married (MSM: $83.9 \%$, FSW: 64.7\%, PWID: $58.7 \%$ ); of note, $7.1 \%$ of MSM were married or cohabitating with women, while $3.4 \%$ of MSM were married or cohabitating with men. Across all three populations, the majority of participants had secondary education or higher (MSM: 83.3\%, FSW: 63.0\%, PWID: 57.2\%). Among MSM participants, 59.9\% were employed while among FSW participants, $23.8 \%$ reported additional employment aside from sex work; employment status was not assessed for PWID. Close to two-thirds of MSM participants and male PWID participants were circumcised, 64.3 and $65.7 \%$ respectively. Most FSW participants reported ever being pregnant (69.2\%).

RDS-weighted aggregate estimates of self-reported symptoms and STIs among MSM, FSW and PWID survey participants are presented in Table 2. Among MSM participants, $5.8 \%$ (95\% CI: 3.4-8.2) reported penile discharge, $6.6 \%$ (95\% CI: $4.0-9.2)$ reported a sore or ulcer near the anus or penis, and 5.0\% (95\% CI: 3.5-6.5) reported a previous STI diagnosis; as such $11.9 \%$ (95\% CI: 7.8-16.0) of MSM participants had a self-reported STI. For FSW participants pooled across the three survey cities, vaginal discharge was reported by $26.7 \%$ (95\% CI: $19.0-34.5$ ), while 9.1\% (95\% CI: 1.6-16.7) reported a sore or ulcer near the vagina and $11.2 \%$ (95\% CI: 7.1-15.2) reported having been previously diagnosed with an STI; there were $33.6 \%$ of FSW participants with self-reported STIs (95\% CI: 26.0 41.3). Among PWID participants - the majority of whom were men (94.9\%) - 13.0\% (95\% CI: 9.6-16.3) reported an abnormal discharge from the vagina, anus or penis, 9.7\% (95\% CI: 6.7-12.6) reported a sore or ulcer near the vagina, penis or anus, and $14.2 \%$ (95\% CI: 11.0-17.4) reported being diagnosed with an STI; self-reported STI was reported by $22.0 \%$ (95\% CI: 17.0-27.0) of participants.

\section{Correlates of STI self-report among MSM}

When controlling for potential confounders in the multiple regression analysis, MSM participants who had been circumcised $(\mathrm{aOR}=1.9,95 \% \mathrm{CI}: 1.3-2.6 ; p<0.001)$, HIV positive (aOR $=2.0,95 \% \mathrm{CI}: 1.2-3.4 ; p=0.009)$, reported illicit drug use $(\mathrm{aOR}=2.2,95 \% \mathrm{CI}: 1.4-3.6 ; p=$ $0.001)$, engaged in receptive anal sex $(\mathrm{aOR}=1.4,95 \% \mathrm{CI}$ : 
Table 1 Unweighted pooled demographic characteristics of Men who have sex with men (MSM), Female sex workers (FSW) and People who inject drugs (PWID), 2010-2014

\begin{tabular}{|c|c|c|c|c|c|c|}
\hline \multirow[t]{2}{*}{ Demographic characteristics } & \multicolumn{2}{|c|}{$\operatorname{MSM}(\boldsymbol{N}=1432)$} & \multicolumn{2}{|c|}{ FSW $(\boldsymbol{N}=1242)$} & \multicolumn{2}{|c|}{ PWID $(\boldsymbol{N}=492)$} \\
\hline & $\mathrm{n}$ & $\%$ & $\mathrm{n}$ & $\%$ & $\mathrm{n}$ & $\%$ \\
\hline \multicolumn{7}{|l|}{ Survey City } \\
\hline Maputo & 496 & 34.6 & 400 & 32.3 & 353 & 71.8 \\
\hline Beira & 583 & 40.7 & 411 & 33.2 & - & - \\
\hline Nampula/Nacala ${ }^{a}$ & 353 & 24.7 & 429 & 34.6 & 139 & 28.3 \\
\hline Male Sex (PWID only) & - & - & - & - & 467 & 94.9 \\
\hline \multicolumn{7}{|l|}{ Age } \\
\hline Median (min-max) & \multicolumn{2}{|c|}{$21(18-59)$} & \multicolumn{2}{|c|}{$21(15-53)$} & \multicolumn{2}{|c|}{$32(18-60)$} \\
\hline 15-24 (FSW), 18-24 (MSM, PWID) & 1134 & 79.2 & 888 & 71.6 & 92 & 18.7 \\
\hline $25+$ & 298 & 20.8 & 352 & 28.4 & 400 & 81.3 \\
\hline \multicolumn{7}{|l|}{ Relationship status } \\
\hline Single or never married & 1198 & 83.9 & 798 & 64.5 & 289 & 58.7 \\
\hline Married/cohabitating (FSW, PWID) & & & 87 & 7.0 & 103 & 20.9 \\
\hline Married/cohabitating, with woman (MSM only) & 102 & 7.1 & - & - & - & - \\
\hline Married/cohabitating with man (MSM only) ${ }^{\mathrm{b}}$ & 48 & 3.4 & - & - & - & - \\
\hline Other (widowed, divorced or separated) & 80 & 5.6 & 352 & 28.5 & 100 & 20.3 \\
\hline \multicolumn{7}{|l|}{ Education Level } \\
\hline No education or Primary level & 238 & 16.7 & 458 & 37.0 & 210 & 42.8 \\
\hline Secondary education or higher & 1191 & 83.3 & 779 & 63.0 & 281 & 57.2 \\
\hline Currently employed (FSW and PWID only) ${ }^{c}$ & 855 & 59.9 & 294 & 23.8 & - & - \\
\hline Circumcised (MSM and male PWID only) & 919 & 64.3 & & & 307 & 65.7 \\
\hline Ever Pregnant (FSW only) & - & _- & 856 & 69.2 & - & - \\
\hline
\end{tabular}

$1.0-2.0 ; p=0.043)$, and reported non-condom use with their last male partner $(\mathrm{aOR}=1.8,95 \% \mathrm{CI}: 1.2-2.5 ; p=$ 0.001 ) had greater odds of STI self-report, as presented in Table 3.

\section{Correlates of STI self-report among FSW}

Table 4 presents the unweighted pooled estimates of risk factors associated with self-reported STIs among FSW. In the multivariable analysis, when controlling for potential confounders, FSW participants living in Beira $(\mathrm{aOR}=$ 2.0, 95\% CI: 1.5-2.8, $p<0.001$ ), currently married $(\mathrm{aOR}=2.0,95 \% \mathrm{CI}: 1.2-3.3, p=0.005)$, having employment aside from sex work $(\mathrm{aOR}=1.5,95 \% \mathrm{CI}$ : $1.1-2.0$, $p=0.007)$, having experienced physical violence $(\mathrm{aOR}=$ 1.5, 95\% CI: $1.1-2.2, p=0.023)$, having experienced sexual violence $(\mathrm{aOR}=2.0,95 \% \mathrm{CI}: 1.3-2.9, p=0.001)$, reporting drug use $(\mathrm{aOR}=4.0,95 \% \mathrm{CI}: 1.5-10.5, p=$ $0.005)$, having access to comprehensive HIV prevention services $(\mathrm{aOR}=1.4,95 \% \mathrm{CI}: 1.0-2.0, p=0.031$ ), reporting non-condom use with last client $(\mathrm{aOR}=1.4,95 \% \mathrm{CI}$ : $1.0-1.9 ; p=0.030$ ), and having had concurrent sexual relationship with a non-client steady partner while engaging in sex work $(\mathrm{aOR}=1.4,95 \% \mathrm{CI}$ : $1.1-1.9, p=$ $0.007)$ had greater odds of STI self-report.

\section{Correlates of STI self-report among PWID}

There were greater odds of self-reported STI among PWID who were living in Nampula/Nacala $(\mathrm{aOR}=4.8$, 95\% CI: $2.3-10.3 ; p<0.001$ ), had access to HIV prevention services $(\mathrm{aOR}=2.3,95 \% \mathrm{CI}: 1.2-4.5 ; p<0.011)$ and reported receiving drugs in exchange for money $(\mathrm{aOR}=$ 2.2, 95\% CI: $1.0-4.7 ; p=0.40$ ), as presented in Table 5.

\section{Discussion}

STI self-report was higher among the KP groups compared to the general population (MSM: $11.9 \%$, FSW: 33.5\%, PWID: $22.0 \%$ vs Men: $5.0 \%$ and Women: $7.0 \%)$. The high-burden of STIs among KPs are consistent with other studies and underscores the importance of integrating STI prevention efforts in KP prevention and treatment services $[21,22]$. This is especially important among FSW where a third of the FSW participants selfreported STI, thus highlighting the generalized risk of STI among this entire population group. 


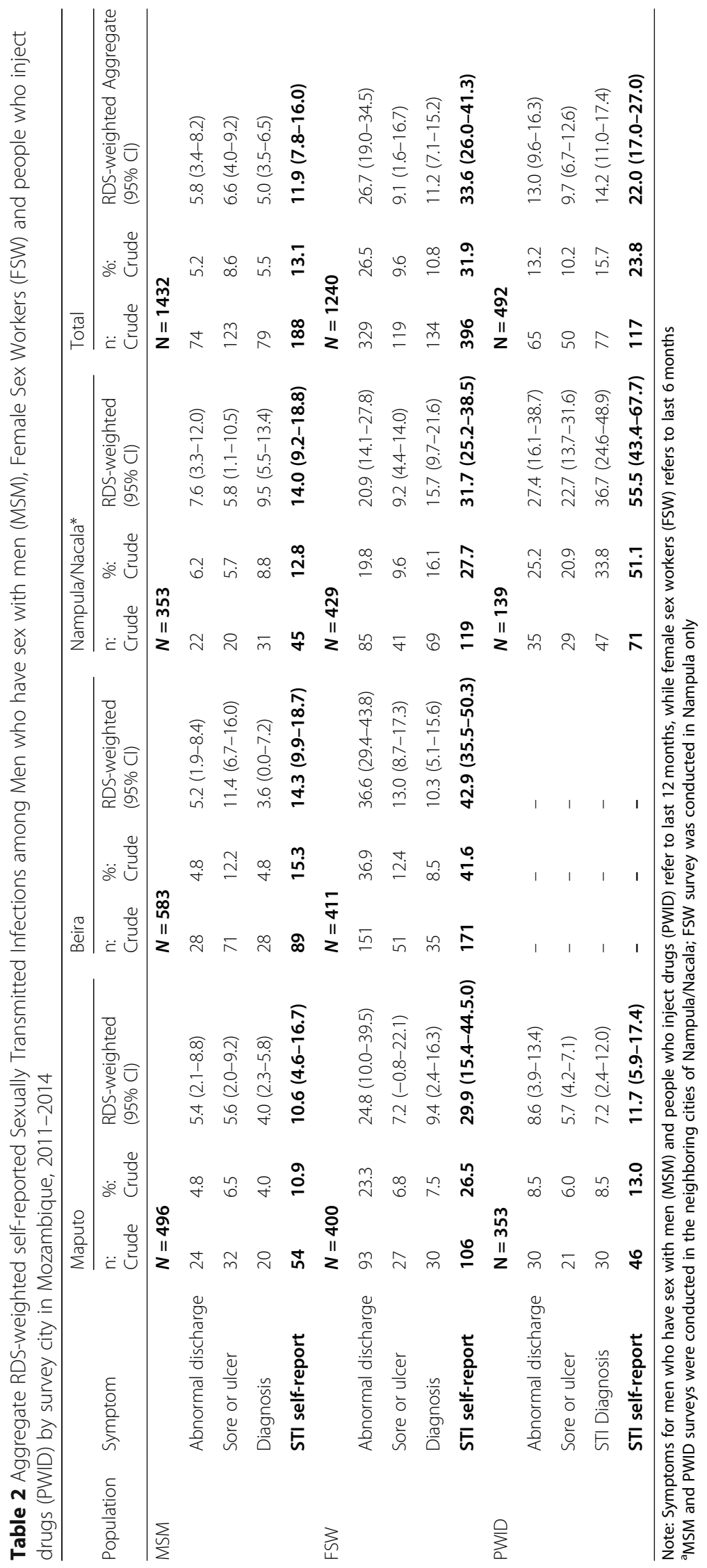


Table 3 Unweighted pooled estimate of risk factors associated with self-reported Sexually Transmitted Infections among Men who have sex with men $(n=1432)$, Mozambique 2012

\begin{tabular}{|c|c|c|c|c|c|c|c|c|}
\hline \multirow[t]{2}{*}{ Variables } & \multicolumn{2}{|l|}{ Prevalence } & \multicolumn{3}{|c|}{ Crude Odds Ratio } & \multicolumn{3}{|c|}{ Adjusted Odds Ratio } \\
\hline & $\mathrm{n} / \mathrm{N}$ & $\%$ & OR & $95 \% \mathrm{Cl}$ & $p$-value & $\mathrm{aOR}$ & $95 \% \mathrm{Cl}$ & $\boldsymbol{p}$-value \\
\hline \multicolumn{9}{|l|}{ Survey City } \\
\hline Maputo & $54 / 496$ & 10.9 & 0.8 & $0.5-1.3$ & 0.104 & & & \\
\hline Beira & $89 / 583$ & 15.3 & 1.2 & $0.8-1.8$ & 0.058 & & & \\
\hline Nampula/Nacala & $45 / 353$ & 12.8 & REF & & & & & \\
\hline \multicolumn{9}{|l|}{ Age (years) } \\
\hline $18-24$ & $127 / 1134$ & 11.2 & REF & & & REF & & \\
\hline 25 and over & $61 / 298$ & 20.5 & 2.0 & $1.5-2.9$ & $<0.001$ & 1.2 & $0.8-1.8$ & 0.395 \\
\hline \multicolumn{9}{|l|}{ Relationship status } \\
\hline Single or never married & $134 / 1198$ & 11.2 & REF & & & REF & & \\
\hline Married/co-habituating, with woman & 29/102 & 28.4 & 3.2 & $2.0-5.0$ & 0.008 & 1.8 & $1.0-3.2$ & 0.273 \\
\hline Married/cohabitating with man $^{a}$ & $9 / 48$ & 18.8 & 1.8 & $0.9-3.9$ & 0.988 & 1.6 & $0.7-3.7$ & 0.672 \\
\hline Other (widowed, divorced or separated) & $16 / 80$ & 20.0 & 2.0 & $1.1-3.5$ & 0.749 & 1.4 & $0.7-2.6$ & 0.893 \\
\hline \multicolumn{9}{|l|}{ Education Level } \\
\hline No education or Primary education & $41 / 238$ & 17.2 & 1.5 & $1.0-2.2$ & 0.043 & 1.2 & $0.8-1.8$ & 0.463 \\
\hline Secondary education or higher & $147 / 1191$ & 12.3 & REF & & & REF & & \\
\hline \multicolumn{9}{|l|}{ Employment Status } \\
\hline Employed & $134 / 855$ & 15.7 & 1.8 & $1.3-2.5$ & $<0.001$ & 1.3 & $0.9-1.8$ & 0.242 \\
\hline Unemployed & $54 / 573$ & 9.4 & REF & & & REF & & \\
\hline \multicolumn{9}{|l|}{ Circumcised } \\
\hline Yes & $99 / 919$ & 10.8 & REF & & & REF & & \\
\hline No & $89 / 510$ & 17.5 & 1.8 & $1.3-2.4$ & $<0.001$ & 1.9 & $1.3-2.6$ & $<0.001$ \\
\hline \multicolumn{9}{|l|}{ HIV infection } \\
\hline Positive & $34 / 114$ & 29.8 & 3.1 & $2.0-4.9$ & $<0.001$ & 2.0 & $1.2-3.4$ & 0.009 \\
\hline Negative & $151 / 1262$ & 12.0 & REF & & & REF & & \\
\hline \multicolumn{9}{|l|}{ Comprehensive prevention services** } \\
\hline Yes & $71 / 467$ & 15.2 & 1.3 & $0.9-1.8$ & 0.111 & & & \\
\hline No & $117 / 962$ & 12.2 & REF & & & & & \\
\hline \multicolumn{9}{|c|}{ Binge drinking ( 6 or more alcoholic drinks per event) } \\
\hline Yes & $77 / 473$ & 16.3 & 1.4 & $1.1-2.0$ & 0.022 & 1.3 & $0.9-1.8$ & 0.199 \\
\hline No & $111 / 912$ & 11.8 & REF & & & REF & & \\
\hline \multicolumn{9}{|l|}{ Illicit drug use } \\
\hline Yes & $30 / 139$ & 21.6 & 2.0 & $1.3-3.1$ & 0.002 & 2.2 & $1.4-3.6$ & 0.001 \\
\hline No & $158 / 1290$ & 12.3 & REF & & & REF & & \\
\hline \multicolumn{9}{|l|}{ Physical violence } \\
\hline Yes & $10 / 51$ & 19.6 & 1.6 & $0.8-3.3$ & 0.170 & & & \\
\hline No & 178/1377 & 12.9 & REF & & & & & \\
\hline \multicolumn{9}{|l|}{ Sexual violence } \\
\hline Yes & $6 / 18$ & 33.3 & 3.4 & $1.3-9.1$ & 0.016 & 1.3 & $0.4-4.3$ & 0.720 \\
\hline No & 181/1409 & 12.9 & REF & & & REF & & \\
\hline \multicolumn{9}{|l|}{ Experienced stigma } \\
\hline Yes & $23 / 129$ & 17.8 & 1.5 & $0.9-2.4$ & 0.101 & & & \\
\hline No & $165 / 1300$ & 12.7 & REF & & & & & \\
\hline
\end{tabular}


Table 3 Unweighted pooled estimate of risk factors associated with self-reported Sexually Transmitted Infections among Men who have sex with men $(n=1432)$, Mozambique 2012 (Continued)

\begin{tabular}{|c|c|c|c|c|c|c|c|c|}
\hline \multirow[t]{2}{*}{ Variables } & \multicolumn{2}{|l|}{ Prevalence } & \multicolumn{3}{|c|}{ Crude Odds Ratio } & \multicolumn{3}{|c|}{ Adjusted Odds Ratio } \\
\hline & $\mathrm{n} / \mathrm{N}$ & $\%$ & $\mathrm{OR}$ & $95 \% \mathrm{Cl}$ & $\boldsymbol{p}$-value & $\mathrm{aOR}$ & $95 \% \mathrm{Cl}$ & $\boldsymbol{p}$-value \\
\hline \multicolumn{9}{|c|}{ Concurrent male and female partner } \\
\hline Yes & 109/736 & 14.8 & 1.4 & $1.0-1.8$ & 0.057 & 1.4 & $1.0-1.9$ & 0.088 \\
\hline No & $79 / 693$ & 11.4 & REF & & & REF & & \\
\hline \multicolumn{9}{|c|}{ Receptive anal sex, with man } \\
\hline Yes & $79 / 523$ & 15.1 & 1.3 & $1.0-1.8$ & 0.098 & 1.4 & $1.0-2.0$ & 0.043 \\
\hline No & 109/906 & 12.0 & REF & & & REF & & \\
\hline \multicolumn{9}{|c|}{ Insertive anal sex, with man } \\
\hline Yes & $163 / 1219$ & 13.4 & 1.1 & $0.7-1.8$ & 0.562 & & & \\
\hline No & $25 / 210$ & 11.9 & REF & & & & & \\
\hline \multicolumn{9}{|c|}{ Condom use at last sexual encounter, with man } \\
\hline No Condom & $114 / 1021$ & 11.2 & 1.8 & $1.3-2.4$ & $<0.001$ & 1.8 & $1.2-2.5$ & 0.001 \\
\hline Yes Condom & $74 / 400$ & 18.0 & REF & & & REF & & \\
\hline \multicolumn{9}{|c|}{ Paid or received sex in exchange for money, with man } \\
\hline Yes & $88 / 636$ & 13.8 & 1.1 & $0.8-1.5$ & 0.507 & & & \\
\hline No & 100/791 & 12.6 & REF & & & & & \\
\hline
\end{tabular}

Notes

a 2 men who have sex with men (MSM) living with men were legally married to a woman

${ }^{* *}$ Comprehensive prevention services refers to peer education \& Information education and communication (IEC) materials

The results from MSM show that STI self- report was associated with receptive (vs insertive) sex, which is consistent with findings from other studies in the region [23]. Other risk factors consistent with the literature included circumcision, which may be a result of decreased perception of risk. Physical and sexual violence were also major risk factors for STI among FSW and the sociocultural dynamics contributing to this vulnerability, such as gender power inequalities, economic disparities, and criminalization of sex work, have been explored previously $[21,24,25]$.

The results suggest that having other work aside from sex work is associated with more risk, however this requires further investigation given that it is contrary to findings among FSW in Uganda where having employment outside of sex work was considered a protective factor [21].

While HIV infection was only significantly associated with STI self-report among MSM [11, 12], the similar modes of sexual transmission necessitate a need for concentrated efforts to encourage safer sexual behaviours, such as condom use. This is especially important when considering the greater odds of STI-self report among FSW reporting non-condom use with their last client and MSM reporting non-condom use with their last male partner. Given the dynamics of bridging populations - a subgroup of people who have sexual contact with both KP and the general population such as MSM married to women, clients of FSW and non-client sexual partners of FSW - non-condom use represents a potential public health risk to the wider population [2]. Access to HIV prevention services was associated with STI-self report among FSW and PWID, although this may be a result of having symptoms which put one in contact with health services.

Our findings draw attention to the intersectionality of key population groups and their risk behaviours. For example, sex work was associated with STI self-report for PWID, while illicit drug use was a risk factor among both FSW and MSM; these overlapping risk profiles were also found in other studies [22, 24]. Our results emphasize that treatment and prevention efforts are limited when only considering the primary risk behaviour of a population while isolating others. Any efforts targeted to KP must adopt a people-centred approach to address overlapping risk behaviours.

Although the sample size of female PWID in our study was too small to perform meaningful analysis $(n=25)$, other studies have pointed to the unique vulnerabilities of female PWID [26]. Qualitative studies among female PWID in Mozambique would provide more information about the gendered nature of risk factors in this group.

Finally, there were greater odds of self-report among FSW residing in Beira and PWID residing in Nampula/ Nacala. Limited resources require the geographic prioritization of efforts based on evidence and require the standardized implementation of quality treatment and prevention services. 
Table 4 Unweighted pooled estimates of risk factors associated with self-reported Sexually Transmitted Infections among Female Sex Workers ( $N=1242)$, Mozambique 2012

\begin{tabular}{|c|c|c|c|c|c|c|c|c|}
\hline \multirow[t]{2}{*}{ Variables } & \multicolumn{2}{|c|}{ Prevalence } & \multicolumn{3}{|c|}{ Crude Odds Ratio } & \multicolumn{3}{|c|}{ Adjusted Odds Ratio } \\
\hline & $\bar{n} / \mathrm{N}$ & (\%) & $\overline{\mathrm{OR}}$ & $95 \% \mathrm{Cl}$ & p-value & $\overline{a O R}$ & $95 \% \mathrm{Cl}$ & $\overline{p \text {-value }}$ \\
\hline \multicolumn{9}{|l|}{ Survey City } \\
\hline Maputo & $106 / 400$ & 26.5 & 0.9 & $0.7-1.3$ & 0.006 & 1.1 & $0.8-1.6$ & 0.126 \\
\hline Beira & $171 / 411$ & 41.6 & 1.9 & $1.4-2.5$ & $<0.001$ & 2.0 & $1.5-2.8$ & $<0.001$ \\
\hline Nampula & $119 / 429$ & 27.7 & REF & & & REF & & \\
\hline \multicolumn{9}{|l|}{ Age (years) } \\
\hline $15-24$ & $279 / 888$ & 31.4 & & & & & & \\
\hline 25 and over & $117 / 352$ & 33.2 & & & & & & \\
\hline \multicolumn{9}{|l|}{ Marital Status } \\
\hline Single or never married & 239/798 & 30.0 & REF & & & REF & & \\
\hline Married/Co-habiting & $42 / 87$ & 48.3 & 2.2 & $1.4-3.4$ & 0.001 & 2.0 & $1.2-3.3$ & 0.005 \\
\hline Other (widowed, divorced or separated) & $115 / 352$ & 32.7 & 1.1 & $0.9-1.5$ & 0.101 & 1.0 & $0.8-1.4$ & 0.079 \\
\hline \multicolumn{9}{|l|}{ Education Level } \\
\hline No education or Primary level education & $154 / 458$ & 33.6 & & & & 1.0 & $0.8-1.4$ & 0.750 \\
\hline Secondary education or higher & $242 / 779$ & 31.1 & & & & & & \\
\hline \multicolumn{9}{|l|}{ Work aside from sex work } \\
\hline Other work & $122 / 294$ & 41.5 & 1.7 & $1.3-2.3$ & $<0.001$ & 1.5 & $1.1-2.0$ & 0.007 \\
\hline No other work & $274 / 943$ & 29.1 & REF & & & REF & & \\
\hline \multicolumn{9}{|c|}{ Binge drinking (6 or more alcoholic drinks per event) } \\
\hline Yes & $125 / 334$ & 37.4 & 1.4 & $1.1-1.8$ & 0.013 & 1.2 & $0.9-1.6$ & 0.153 \\
\hline No & 270/901 & 30.0 & REF & & & REF & & \\
\hline \multicolumn{9}{|l|}{ Physical Violence ${ }^{a}$} \\
\hline Yes & 78/172 & 45.4 & 2.0 & $1.4-2.7$ & $<0.001$ & 1.5 & $1.1-2.2$ & 0.023 \\
\hline Never & $316 / 1061$ & 29.8 & REF & & & REF & & \\
\hline \multicolumn{9}{|l|}{ Sexual Violence ${ }^{a}$} \\
\hline Yes & $71 / 138$ & 51.5 & 2.5 & $1.8-3.6$ & $<0.001$ & 2.0 & $1.3-2.9$ & 0.001 \\
\hline No & $325 / 1098$ & 29.6 & REF & & & REF & & \\
\hline \multicolumn{9}{|l|}{ Illicit drug use } \\
\hline Yes & $16 / 24$ & 66.7 & 4.4 & $1.9-10.3$ & $<0.001$ & 4.0 & $1.5-10.5$ & 0.005 \\
\hline No & $380 / 1213$ & 31.3 & REF & & & REF & & \\
\hline \multicolumn{9}{|l|}{ HIV infection } \\
\hline Positive & $123 / 341$ & 36.1 & 1.3 & $1.0-1.7$ & 0.060 & 1.2 & $0.9-1.6$ & 0.253 \\
\hline Negative & 273/896 & 30.5 & REF & & & REF & & \\
\hline \multicolumn{9}{|l|}{ Comprehensive prevention services ${ }^{* *}$} \\
\hline Yes & $80 / 207$ & 38.7 & 1.4 & $1.0-1.9$ & 0.025 & 1.4 & $1.0-2.0$ & 0.031 \\
\hline No & $316 / 1030$ & 30.7 & REF & & & REF & & \\
\hline \multicolumn{9}{|l|}{ Condom use with last client } \\
\hline No condom & $123 / 316$ & 38.9 & 1.5 & $1.2-2.0$ & 0.002 & 1.4 & $1.0-1.9$ & 0.030 \\
\hline Condom & $271 / 919$ & 29.5 & REF & & & REF & & \\
\hline \multicolumn{9}{|l|}{ Concurrent stable romantic partner \& sex work } \\
\hline Yes & $184 / 485$ & 37.9 & 1.6 & $1.2-2.0$ & $<0.001$ & 1.4 & $1.1-1.9$ & 0.007 \\
\hline No & 210/745 & 28.2 & REF & & & REF & & \\
\hline
\end{tabular}

**Comprehensive prevention services refers to peer education \& Information education and communication (IEC) materials 
Table 5 Unweighted pooled estimate of risk factors associated with self-reported syndromic STIs among People Who Inject Drugs ( $N=492)$, Mozambique 2014

\begin{tabular}{|c|c|c|c|c|c|c|c|c|}
\hline \multirow[t]{2}{*}{ Variables } & \multicolumn{2}{|c|}{ Prevalence } & \multicolumn{3}{|c|}{ Crude Odds Ratio } & \multicolumn{3}{|c|}{ Adjusted Odds Ratio } \\
\hline & $\mathrm{n} / \mathrm{N}$ & $(\%)$ & $\overline{O R}$ & $95 \% \mathrm{Cl}$ & $\overline{p \text {-value }}$ & $\mathrm{aOR}$ & $95 \% \mathrm{Cl}$ & $\boldsymbol{p}$-value \\
\hline \multicolumn{9}{|l|}{ Survey City } \\
\hline Maputo & $46 / 353$ & 13.0 & 7.0 & $4.4-11.0$ & $<0.001$ & REF & & \\
\hline Nampula/Nacala & $71 / 139$ & 51.1 & & & & 4.8 & $2.3-10.3$ & $<0.001$ \\
\hline \multicolumn{9}{|l|}{ Sex } \\
\hline Female & $6 / 25$ & 24.0 & 1.0 & $0.4-2.6$ & 0.979 & 1.4 & $0.4-5.0$ & 0.576 \\
\hline Male & $111 / 467$ & 23.8 & REF & & & REF & & \\
\hline \multicolumn{9}{|l|}{ Age (years) } \\
\hline $18-24$ & $32 / 92$ & 34.8 & 2.0 & $1.2-3.2$ & 0.007 & 1.0 & $0.5-2.3$ & 0.955 \\
\hline 25 and over & $85 / 400$ & 21.3 & REF & & & REF & & \\
\hline \multicolumn{9}{|l|}{ Marital Status } \\
\hline Single or never married & $54 / 289$ & 18.7 & REF & & & & & \\
\hline Married/Co-habiting & $34 / 103$ & 33.0 & 2.1 & $1.3-3.6$ & 0.056 & 1.6 & $0.8-3.4$ & 0.947 \\
\hline Other (widowed, divorced or separated) & $29 / 100$ & 29.0 & 1.8 & $1.1-3.0$ & 0.448 & 2.4 & $1.2-5.0$ & 0.055 \\
\hline \multicolumn{9}{|l|}{ Education Level } \\
\hline No education or Primary level education & $37 / 210$ & 17.6 & REF & & & & & \\
\hline Secondary education or higher & $80 / 281$ & 28.5 & 1.9 & $1.2-2.9$ & 0.006 & 0.9 & $0.5-1.7$ & 0.849 \\
\hline \multicolumn{9}{|l|}{ Circumcised (Males only, $n=193$ ) } \\
\hline Yes & $89 / 307$ & 29.0 & 2.6 & $1.5-4.3$ & $<0.001$ & & & \\
\hline No & $22 / 160$ & 13.8 & REF & & & & & \\
\hline \multicolumn{9}{|l|}{ HIV infection } \\
\hline Positive & $44 / 204$ & 21.6 & 0.8 & $0.5-1.2$ & 0.222 & 1.6 & $0.8-3.0$ & 0.197 \\
\hline Negative & $64 / 241$ & 26.6 & REF & & & REF & & \\
\hline \multicolumn{9}{|l|}{ Comprehensive prevention services** } \\
\hline Yes & $41 / 78$ & 52.6 & 4.9 & $3.0-8.2$ & $<0.001$ & 2.3 & $1.2-4.5$ & 0.011 \\
\hline No & $76 / 414$ & 18.4 & REF & & & REF & & \\
\hline \multicolumn{9}{|l|}{ Phyisical Violence $^{\mathrm{a}}$} \\
\hline Yes & $22 / 77$ & 28.6 & 1.3 & $0.8-2.3$ & 0.283 & & & \\
\hline Never & $95 / 415$ & 22.9 & REF & & & & & \\
\hline \multicolumn{9}{|l|}{ Sexual Violence $\mathrm{e}^{\mathrm{a}, \mathrm{b}}$} \\
\hline Yes & $1 / 6$ & 16.7 & 0.6 & $0.1-5.5$ & 0.684 & & & \\
\hline No & $116 / 486$ & 23.9 & REF & & & & & \\
\hline \multicolumn{9}{|l|}{ Experienced stigma ${ }^{a}$} \\
\hline Yes & $39 / 84$ & 27.4 & 3.8 & $2.3-6.2$ & $<0.001$ & 1.0 & $0.5-1.9$ & 0.974 \\
\hline No & $72 / 387$ & 14.7 & REF & & & REF & & \\
\hline \multicolumn{9}{|l|}{ Condom use at last sexual encounter } \\
\hline Yes & $36 / 173$ & 20.8 & 1.7 & $0.9-3.3$ & 0.957 & 1.0 & $0.4-2.4$ & 0.888 \\
\hline No & $67 / 214$ & 31.3 & 2.9 & $1.6-5.5$ & $<0.001$ & 1.1 & $0.5-2.7$ & 0.726 \\
\hline Not sexually active & $14 / 104$ & 13.5 & REF & & & REF & & \\
\hline \multicolumn{9}{|l|}{ Received drugs in exchange for sex } \\
\hline Yes & $27 / 67$ & 40.3 & 2.5 & $1.5-4.3$ & 0.001 & 2.2 & $1.0-4.7$ & 0.040 \\
\hline No & $90 / 424$ & 21.2 & REF & & & REF & & \\
\hline
\end{tabular}

a Last $12 \mathrm{~m}$ (PWID)

${ }^{b}$ Fishers exact test

${ }^{*}$ Comprehensive prevention services refers to peer education \& Information education and communication (IEC) materials 
Since the implementation of these BBS surveys, the National HIV Program has scaled up prevention, care and treatment efforts for KPs in Mozambique. In 2016, National Guidelines were published that aimed to integrate HIV prevention and treatment services for KPs into the health sector [27]. These included the creation of standardized package of services for KPs with structural, biomedical and behavioural interventions, including STI screening, diagnosis and treatment. The guidelines commit to offering evidence-based quality services with a people-centred approach free from stigma and discrimination. The guidelines also aimed to strengthen the linkage between community and clinical services to ensure HIV testing among these hard to reach populations. The importance of STI prevention and control among KP was further outlined in the 2018-2021 National Strategic Plan for the Prevention and Control of STIs [6]. Future BBS surveys will be able to assess the extent KPs engagement with the health system, experiences of stigma and STI self-report.

Although this is the first analysis of risk factors associated with STIs among MSM, FSW and PWID in Mozambique, there are several limitations to consider. First, the reliance of self-reported STI symptoms, rather than laboratory testing of common and treatable STIs such as syphilis, chlamydia, and gonorrhoea, could have potentially underestimated STI prevalence by excluding asymptomatic cases. In addition, symptoms such as, discharge, may not necessarily have been the result of an STI. Second, not all survey measures were included in the three surveys (e.g. stigma/discrimination), thus it is not possible to compare risk factors across the different population groups. Additionally, the survey is also subject to the limitations to the survey design such as social desirability, interviewer and recruitment bias. Similar to other cross-sectional surveys, it is also not possible to assess temporality. For example, it is not possible to determine if having access to health services brought PWID and FSW in contact with STI diagnosis or if perhaps having an STI symptom may have caused one to seek out services. Finally, the analysis pooled results from across the survey cities thus severing social networks and chains. As a result, these findings need to be interpreted with caution and cannot be generalized to the full MSM, FSW and PWID in the survey cities nor to KP in Mozambique. Despite the limitations, however, the results of the analysis point to the high burden of STIs among key population groups in Mozambique and provide the evidence needed to advocate for comprehensive and integrated policies and health systems approaches to improve STI screening and case management among high-risk groups in Mozambique.

\section{Conclusion}

The high burden of STIs in KP highlights the need for integrated HIV and STI prevention, outreach and treatment services that address the overlapping risk profiles of individuals; this is specifically relevant for harm reduction interventions targeted to PWID that must also include STI screening and promote condom use. Future survey and surveillance studies should consider including laboratory testing of STIs in order to identify and treat asymptomatic cases. Finally, a robust public health response would include the creation of a national STI surveillance system, for better screening and diagnostic procedures. Monitoring the prevalence of STIs, especially among $\mathrm{KP}$, must be seen as an important element of any efforts toward HIV epidemic control.

\section{Abbreviations}

BBS: Biological and behavioral Survey; KP: Key populations; FSW: Female sex workers; HIV: Human immunodeficiency virus; MSM: Men who have sex with men (MSM); PWID: People who inject drugs (PWID); RDS: Respondent-driven sampling; RDS-A: RDS-Analyst; STI: Sexually transmitted Infection; WHO: World Health Organization

\section{Acknowledgements}

The study team acknowledges the immense contributions of the Mozambican BBS Technical Working Group, and all who have contributed to the successful implementation of the MSM, FSW and PWID surveys in Mozambique.

\section{Authors' contributions}

All authors reviewed and provided edits and comments on manuscript drafts. In addition, authors had the following responsibilities: MB had full access to the data, conducted data analysis, drafted and revised the manuscript, and takes responsibility for the integrity of the data, accuracy of the data analysis and contents of this article; CC was involved in BBS survey implementation, laboratory testing and data interpretation. IS was involved in BBS survey recruitment, data management and data analysis; $\mathrm{RH}$ was responsible for overall study design and for overseeing data analysis; CSB was involved in BBS recruitment and implementation activities and provided scientific oversight; DCL and NC were involved in survey design and provided overall scientific oversight of the study; JS was responsible for all BBS clinical aspects, implementation and overall scientific oversight; HFR designed the study, was the BBS principal investigator, and was involved in recruitment, scientific oversight and data analysis. EF, MT and LS provided critical revision and final approval of the manuscript.

\section{Funding}

This research has been supported by the President's Emergency Plan for AIDS Relief (PEPFAR) through the Centers for Disease Control and Prevention (CDC) under the terms of the Cooperative Agreement \#U2GPS001468. The findings and conclusions in this report are those of the author(s) and do not necessarily represent the official position of the CDC. CDC investigators did not participate in the collection of data, but they provided general oversight of protocol development, data analysis, and drafting of the survey reports.

\section{Availability of data and materials}

The dataset analysed for the current study are fully available from the Data Management Unit of the Mozambique National Institute of Health (INS) data repository for researchers who meet the criteria for access to confidential data following the submission of a concept note. For information, please visit: www.ins.gov.mz or contact: secretaria@ins.gov.mz.

Ethics approval and consent to participate

All study protocols were approved by the Mozambican National Bioethics Committee for Health, by the Committee on Human Research at the University of California at San Francisco, and by the Division of Global HIV/ 
AIDS of the U.S. Centers for Disease Control and Prevention, Atlanta. For all participants, written informed consent was obtained.

\section{Consent for publication}

Not Applicable.

\section{Competing interests}

The authors declare that they have not competing interests.

\section{Author details}

${ }^{1}$ Faculty of Medicine and Health Sciences, Ghent University, Ghent, Belgium. ${ }^{2}$ National Institute of Health, Maputo, Mozambique. ${ }^{3}$ National STI-HIV/AIDS Control Program, Ministry of Health, Maputo, Mozambique. ${ }^{4}$ School of Public Health, Rutgers University, Piscataway, NJ, USA. ${ }^{5}$ ICAP, Columbia University, Pretoria, South Africa. ${ }^{6}$ Department of Obstetrics and Gynaecology, Aga Khan University, Nairobi, Kenya. ${ }^{7}$ Department of Population Health, Aga Khan University, Nairobi, Kenya. ${ }^{8}$ Department of Epidemiology and Preventive Medicine, Monash University, Melbourne, Australia. ${ }^{9}$ Burnet Institute, Melbourne, Australia.

Received: 11 May 2020 Accepted: 20 July 2020

Published online: 27 August 2020

\section{References}

1. World Health Organization. Global Health Sector Strategy on Sexually Transmitted Infections: 2016-2021. 2016. Available from: https://www.who. int/publications/i/item/WHO-RHR-16.09. [cited 2020 Feb 6].

2. World Health Organization. Prevention and treatment of HIV and other sexually transmitted infections for sex workers in low- and middle-Income countries: recommendations for a public health approach. 2012. Available from: http://www.ncbi.n/m.nih.gov/books/NBK304116/. [cited 2020 Feb 8].

3. World Health Organization. Consolidated guidelines on HIV prevention, diagnosis, treatment and care for key populations. 2014. Available from: http://apps.who.int/iris/bitstream/10665/128048/1/9789241507431_eng.pdf. [cited 2017 Feb 2].

4. Ministério da Saúde, Instituto Nacional de Estatística, ICF Macro. Inquérito de Indicadores de Imunização, Malária e HIV/SIDA em Moçambique (IMASIDA), 2015. 2018. Available from: https://dhsprogram.com/pubs/pdf/ AIS12/AIS12.pdf. [cited 2020 Jun 6].

5. World Health Organization. Strategies and laboratory methods for strengthening surveillance of sexually transmitted infection, 2012. Geneva: World Health Organization; 2012. Available from: http://apps.who.int/iris/ bitstream/10665/75729/1/9789241504478_eng.pdf. [cited 2019 Apr 11].

6. Ministério da Saúde. Plano Estratégico de Acção para a Prevenção e Controlo das Infecções de Transmissão Sexual, 2018-2021. 2018. Available from: http://www.misau.gov.mz/index.php/planos-estrategicos-dohiv?download=171: plano-estrategico-de-accao-prevencao-e-controlo-dasits-2018-2021. [cited 2020 Apr 1].

7. Conselho de Ministros. Plano Estratégico Nacional de Resposta ao HIV e SIDA, 2010-2014 [Internet]. 2010. Available from: https://www.lo.org/ wcmsp5/groups/public/---ed_protect/---protrav/---ilo_aids/documents/ legaldocument/wcms_172584.pdf. [cited 2020 Feb 3].

8. Conselho Nacional de Combate ao HIV e SIDA (CNCS). Plano Estratégico Nacional de Resposta Ao HIV e SIDA, 2015-2019. Maputo, Mozambique; 2015. Available from: http://cncs.co.mz/wp-content/uploads/2016/02/PENIV-2015-2019-Vers\%C3\%A3o-Aprovada-pelo-Conselho-de-Ministros-1.pdf. [cited 2020 Feb 3].

9. Semá Baltazar C, Horth R, Boothe M, Sathane I, Young P, Chitsondzo Langa D, et al. High prevalence of HIV, HBsAg and anti-HCV positivity among people who injected drugs: results of the first bio-behavioral survey using respondent-driven sampling in two urban areas in Mozambique. BMC Infect Dis. 2019 Dec;19(1):1022.

10. Augusto Â, Young PW, Horth RZ, Inguane C, Sathane I, Ngale K, et al. High burden of HIV infection and risk behaviors among female sex Workers in Three Main Urban Areas of Mozambique. AIDS Behav. 2016;20(4):799-810.

11. Nalá R, Cummings B, Horth R, Inguane C, Benedetti M, Chissano M, et al. Men who have sex with men in Mozambique: identifying a hidden population at high-risk for HIV. AIDS Behav. 2015;19(2):393-404.

12. Instituto Nacional de Saúde, Centers for Disease Control and Prevention, University of California, San Francisco, Pathfinder International, I-TECH. The Integrated Biological and Behavioral Survey Among Men Who Have Sex with Men, Mozambique, 2011. 2012. Available from: https:// globalhealthsciences.ucsf.edu/sites/globalhealthsciences.ucsf.edu/files/pub/ ibbs-msm-final-report.pdf. [cited 2020 Jul 31]. .

13. Instituto Nacional de Saúde, Centers for Disease Control and Prevention, University of California, San Francisco, Pathfinder International, I-TECH. The Integrated Biological and Behavioral Survey Among Female Sex Workers, Mozambique, 2011-2012. 2012. Available from: https://ins.gov.mz/node/56. [cited 2020 Jul 31].

14. Ministério da Saúde, Instituto Nacional de Saúde. The Mozambique Integrated Biological and Behavioral Survey Among People Who Inject Drugs, 2014 [Internet]. Maputo, Mozambique; 2017. Available from: https:// globalhealthsciences.ucsf.edu/sites/globalhealthsciences.ucsf.edu/files/pub/ pwid-final-report-20180329.pdf. [cited 2020 Jul 31].

15. Heckathorn DD. Respondent-Driven Sampling II: Deriving valid population estimates from chain-referral samples of hidden populations. Soc Probl. 2002;49(1):11-34.

16. Salganik MJ, Heckathorn DD. Sampling and estimation in hidden populations using respondent-driven Sampling. Sociol Methodol. 2004;34(1): $193-240$.

17. Family Health International. Behavioral Surveillance Surveys: Guidelines for Repeated Behavioral Surveys in Populations at Risk of HIV [Internet]. 2000 [cited 2020 Jun 28]. Available from: https://www.who.int/hiv/strategic/en/ bss_fhi2000.pdf?ua=1.

18. World Health Organization. Core Questions in eQuestionnaires [Internet] 2017 [cited 2020 Jun 28]. Available from: https://www.who.int/hiv/pub/ guidelines/CoreQuestionsineQuestionnaires.pdf?ua=1.

19. Handcock MS, Fellows IE, Gile KJ. RDS Analyst: Software for the Analysis of Respondent-Driven Sampling Data [Internet]. Vol. 0.64. Available from: http://hpmrg.org

20. Volz E, Heckathorn DD. Probability Based Estimation Theory for Respondent Driven Sampling. J Official Statistics. 2008;24(1):79-97.

21. Hladik W, Baughman AL, Serwadda D, Tappero JW, Kwezi R, Nakato ND, et al. Burden integrated Biologican and behavioral survey among men who have sex integrated Biologican and behavioral survey among men who have sex and characteristics of HIV infection among female sex workers in Kampala, Uganda - a respondent-driven sampling survey. BMC Public Health. 2017;17(1):565.

22. Kim EJ, Hladik W, Barker J, Lubwama G, Sendagala S, Ssenkusu JM, et al. Sexually transmitted infections associated with alcohol use and HIV infection among men who have sex with men in Kampala, Uganda. Sexually Transmitted Infect. 2016;92(3):240-5.

23. Keshinro B, Crowell TA, Nowak RG, Adebajo S, Peel S, Gaydos CA, et al. High prevalence of HIV, chlamydia and gonorrhoea among men who have sex with men and transgender women attending trusted community centres in Abuja and Lagos, Nigeria. J Int AIDS Soc. 2016;19(1):21270.

24. Musyoki H, Kellogg TA, Geibel S, Muraguri N, Okal J, Tun W, et al. Prevalence of HIV, sexually transmitted infections, and risk Behaviours among female sex Workers in Nairobi, Kenya: results of a respondent driven Sampling study. AIDS Behav. 2015;19(S1):46-58.

25. Ngale K, Cummings B, Horth R. Unseen, unheard and unprotected: prevalence and correlates of violence among female sex workers in Mozambique. Culture, Health \& Sexuality. 2019;21(8):898-913.

26. Lambdin BH, Bruce RD, Chang O, Nyandindi C, Sabuni N, Zamudio-Haas S, et al. Identifying Programmatic Gaps: Inequities in Harm Reduction Service Utilization among Male and Female Drug Users in Dar es Salaam, Tanzania. Vermund SH, editor. PLoS ONE. 2013;8(6):e67062.

27. Ministério da Saúde. Directriz para Integração dos Serviços de Prevenção, Cuidados e Tratamento em HIV e SIDA para a População Chave no Sector da Saúde [Internet]. Direçcão Nacional de Assisténcia Médica, PNC ITS-HIV/ SIDA; 2016. Available from: http://www.misau.gov.mz/index.php/directrizesnacionais. [cited 2019 Oct 17].

\section{Publisher's Note}

Springer Nature remains neutral with regard to jurisdictional claims in published maps and institutional affiliations. 